\title{
Comparison between two newborn screening strategies for cystic fibrosis in Argentina: IRT/IRT vs. IRT/PAP
}

\author{
Alejandro Teper ${ }^{1}$, Fernando Smithius ${ }^{2}$, Viviana Rodriguez ${ }^{1}$, Orlando Salvaggio ${ }^{1}$, Gustavo \\ Maccallini², Claudio Aranda ${ }^{2}$, Silvina Lubovich ${ }^{3}$, Silvina Zaragoza ${ }^{3}$, and Facundo \\ Garcia-Bournissen ${ }^{4}$ \\ ${ }^{1}$ El Hospital de Niños Ricardo Gutierrez \\ ${ }^{2}$ Hospital General de Agudos Carlos G Durand \\ ${ }^{3}$ El Hospital de Ninos Ricardo Gutierrez \\ ${ }^{4}$ University of Western Ontario Schulich School of Medicine and Dentistry
}

June 3, 2020

\begin{abstract}
Background: Benefits of early Cystic Fibrosis (CF) detection using newborn screening (NBS) lead to widespread use in NBS programs. Since 2002, a two-stage immunoreactive trypsinogen (IRT/IRT) screening strategy has been used as CFNBS method in all public maternities in the City of Buenos Aires, Argentina. However, novel screening strategies may be more efficient. The aim of the study is to prospectively compare two CFNBS strategies, IRT/IRT and IRT/PAP (pancreatitis-associated protein). Methods: A two-year prospective study was performed. IRT was measured in dried blood samples collected 48-72 hours after birth. When IRT value was abnormal, PAP was determined, and a second visit was scheduled to obtain another sample for IRT before 25 days of life. Newborns with a positive CFNBS were referred for confirmatory sweat test. Results: There were 69,827 births in the City of Buenos Aires during the period studied; 918 (1.31\%) had an abnormal IRT. A total of 207 children $(22.5 \%)$ failed to return for the second IRT, but only two PAP $(0.2 \%)$ were not performed. IRT/IRT was more likely to lead to a referral for sweat testing than IRT/PAP (OR 2.3 [95\% CI 1.8;2.9], p<0.001). Sensitivity, specificity, positive predictive value, and negative predictive value were: $80 \%$ and $100 \%, 86.5 \%$ and $82.6 \%, 4.04 \%$ and $4.2 \%, 99.84 \%$ and $100 \%$ for IRT/IRT and IRT/PAP strategies, respectively. Conclusion: The IRT/PAP strategy is more sensitive than IRT/IRT; it avoids a second appointment and the need of unnecessary sweat testing, and decreases loss to follow up in our population.
\end{abstract}

Hosted file

Comparison between two CFNBS strategies in Argentina Text.docx available at https: //authorea.com/users/329396/articles/456529-comparison-between-two-newborn-screeningstrategies-for-cystic-fibrosis-in-argentina-irt-irt-vs-irt-pap

\section{Hosted file}

Comparison between two CFNBS Figures and Tables.docx available at https://authorea.com/users/ 329396/articles/456529-comparison-between-two-newborn-screening-strategies-for-cysticfibrosis-in-argentina-irt-irt-vs-irt-pap 